CARNETS OE Carnets de géographes

GÉOGRAPHES.

$5 \mid 2013$

Géographie humanimale

\title{
Penser avec le corps
}

comment une panthère a transformé ma thèse

Jean Estebanez

\section{OpenEdition}

Journals

Édition électronique

URL : http://journals.openedition.org/cdg/1077

DOI : $10.4000 /$ cdg. 1077

ISSN : 2107-7266

Éditeur

UMR 245 - CESSMA

Référence électronique

Jean Estebanez, «Penser avec le corps », Carnets de géographes [En ligne], 5 | 2013, mis en ligne le 11 mai 2017, consulté le 20 avril 2019. URL : http://journals.openedition.org/cdg/1077 ; DOI : 10.4000/ cdg. 1077

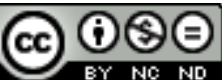

La revue Carnets de géographes est mise à disposition selon les termes de la Licence Creative Commons Attribution - Pas d'Utilisation Commerciale - Pas de Modification 4.0 International. 


\section{PENSER AVEC LE CORPS \\ Comment une panthère $a$ transformé ma thèse}

JEAN ESTEBANEZ

Lab'Urba

Université Paris-Est Créteil

jean.estebanez@u-pec.fr

C'est le milieu de l'après-midi, lors de ma deuxième journée de stage au zoo de Pont Scorff, pendant l'été $2007^{1}$. J'accompagne Stéphanie, une soigneuse d'une vingtaine d'années, dans sa tournée quotidienne de nettoyage des enclos et de distribution de nourriture. Occupé à suivre les étapes de mon protocole de recherche, que je commence à bien connaître, je la photographie dans son travail en lui demandant de m'expliquer ce qu'elle fait. Je note consciencieusement ses réponses en l'encourageant à me parler de son parcours scolaire et professionnel, des raisons et des contingences qui l'ont amenée ici ; bref, je cherche à dresser son portait social (Estebanez, 2010).

Nous abordons le secteur des félins par l'enclos d'une panthère, que je n'avais guère remarquée les jours passés, tant elle se tenait en retrait, dans un renfoncement de son habitat. II faut se rendre dans le réduit à l'arrière de la cage, là où la panthère passe la nuit et prend son repas. II est nettoyé quotidiennement, avant qu'on y dépose des quartiers de viande. Le public n'y accède jamais et n'en soupçonne pas même l'existence, puisqu'il est masqué par la déclivité du terrain.

Nous abandonnons donc le sentier principal, où circulent les visiteurs, pour emprunter une volée de marches mal fixées au flanc de la colline. J'abandonne un instant mes questions et referme mon carnet de notes pour me concentrer sur la pente.

Arrivés derrière l'enclos, Stéphanie contourne une série de câbles, ouvre une porte et me glisse quelques recommandations :

«Alors, quand tu rentres, tu te plaques bien contre le mur [...] fais attention aux fils, c'est les commandes de la porte, si tu tires, ça ouvre... je ne veux pas mourir »

\footnotetext{
${ }^{1}$ C'est une période pendant laquelle j'effectue les premiers terrains dans le cadre de ma thèse (Estebanez, 2010). Je passe en particulier cet été dix jours au zoo de Pont-Scorff, une semaine à Zoodyssée, à proximité de Niort et deux semaines à la Ménagerie du Jardin des Plantes de Paris. Je cherche alors à comprendre le fonctionnement de l'institution et de ses acteurs, les modèles sur lesquels elle s'appuie (le découpage du monde ; l'exotisme ; la mise en scène du sauvage) et à en suivre le public.
} 
Bon, voilà qui est engageant... Je suis loin d'être rassuré. Le réduit, qui doit faire moins de deux mètres de profondeur, est séparé du reste de l'enclos par une grille simple, mince et au maillage lâche.

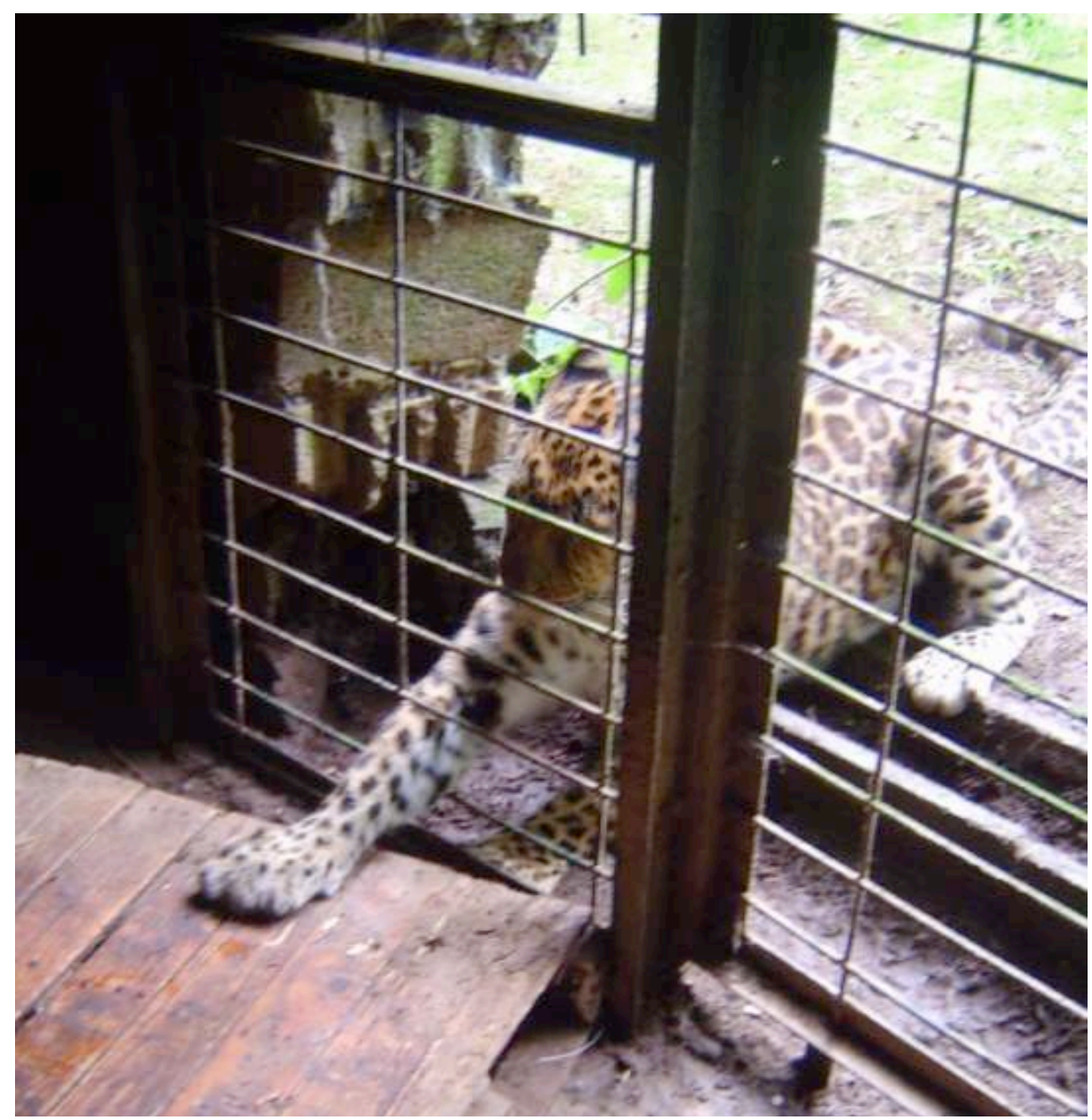

Figure 1 : Une panthère au zoo de Pont Scorff, 27 juillet 2007

Nous entendant arriver, la panthère vient immédiatement s'installer devant la trappe par laquelle elle pourra entrer quelques instants plus tard. Elle rugit en nous regardant, puis passe une patte à l'intérieur du réduit, agrippe la palette en bois qui lui sert de couchage, la soulève et la fracasse contre la grille.

Stéphanie, qui a dû en voir d'autres, commence à balayer le réduit. Pour ma part, je me colle contre la paroi et ne bouge plus. S'engage un échange entre elle et la panthère, par l'intermédiaire du balai que celle-ci chasse, dès qu'il passe à portée de patte. Stéphanie balaye plus longuement qu'il ne serait nécessaire, la panthère ne brise pas l'ustensile : elles jouent ensemble. 


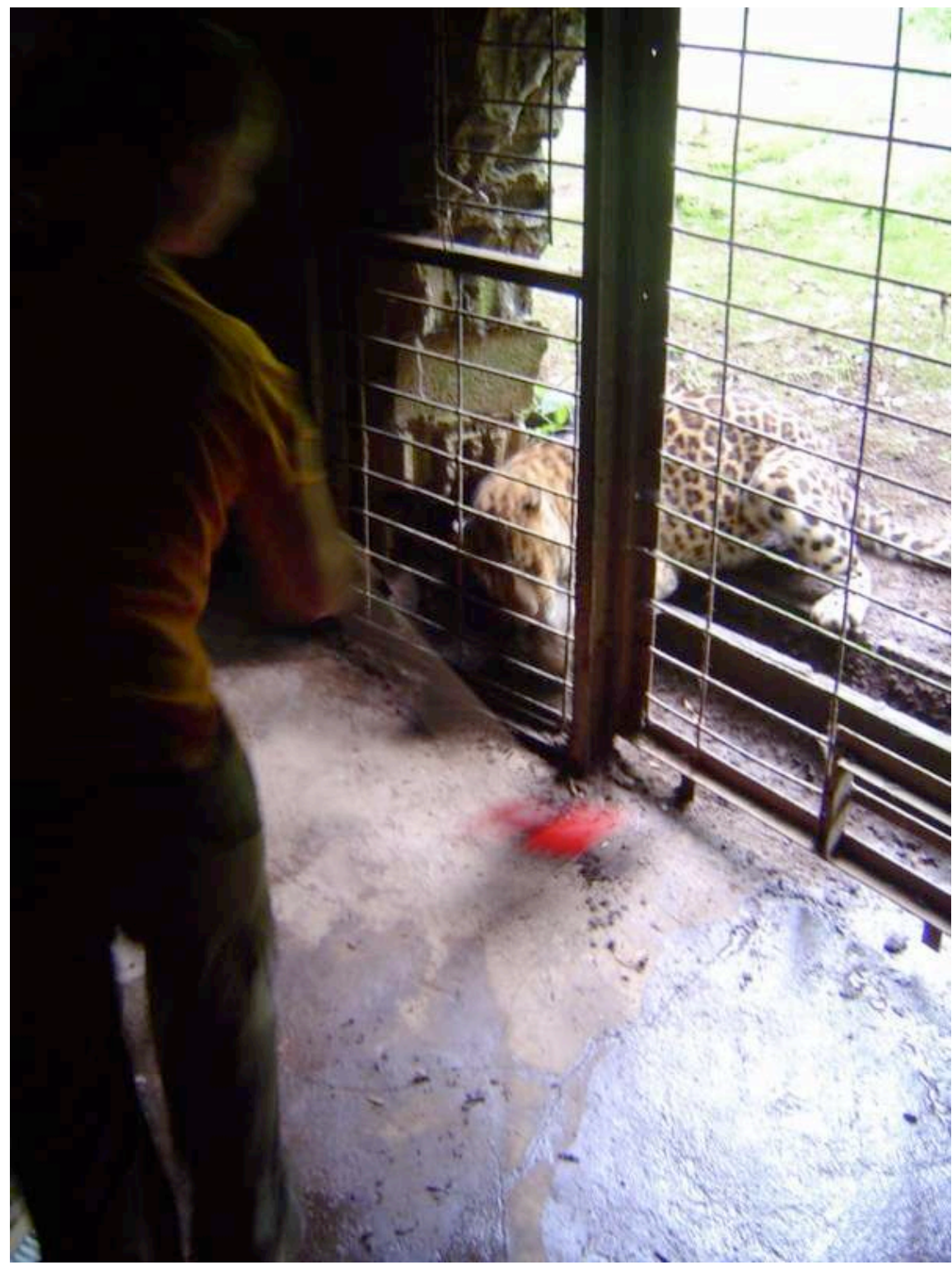

Figure 2 : Le jeu du balai, zoo de Pont Scorff, 27 juillet 2007

Pendant ce temps, je m'installe avec de grandes précautions dans la partie gauche de la cage pour prendre un portrait de la panthère. Je m'accroupis pour me mettre à son niveau, dos au mur. Immédiatement, la panthère abandonne le balai pour se concentrer sur la porte. 


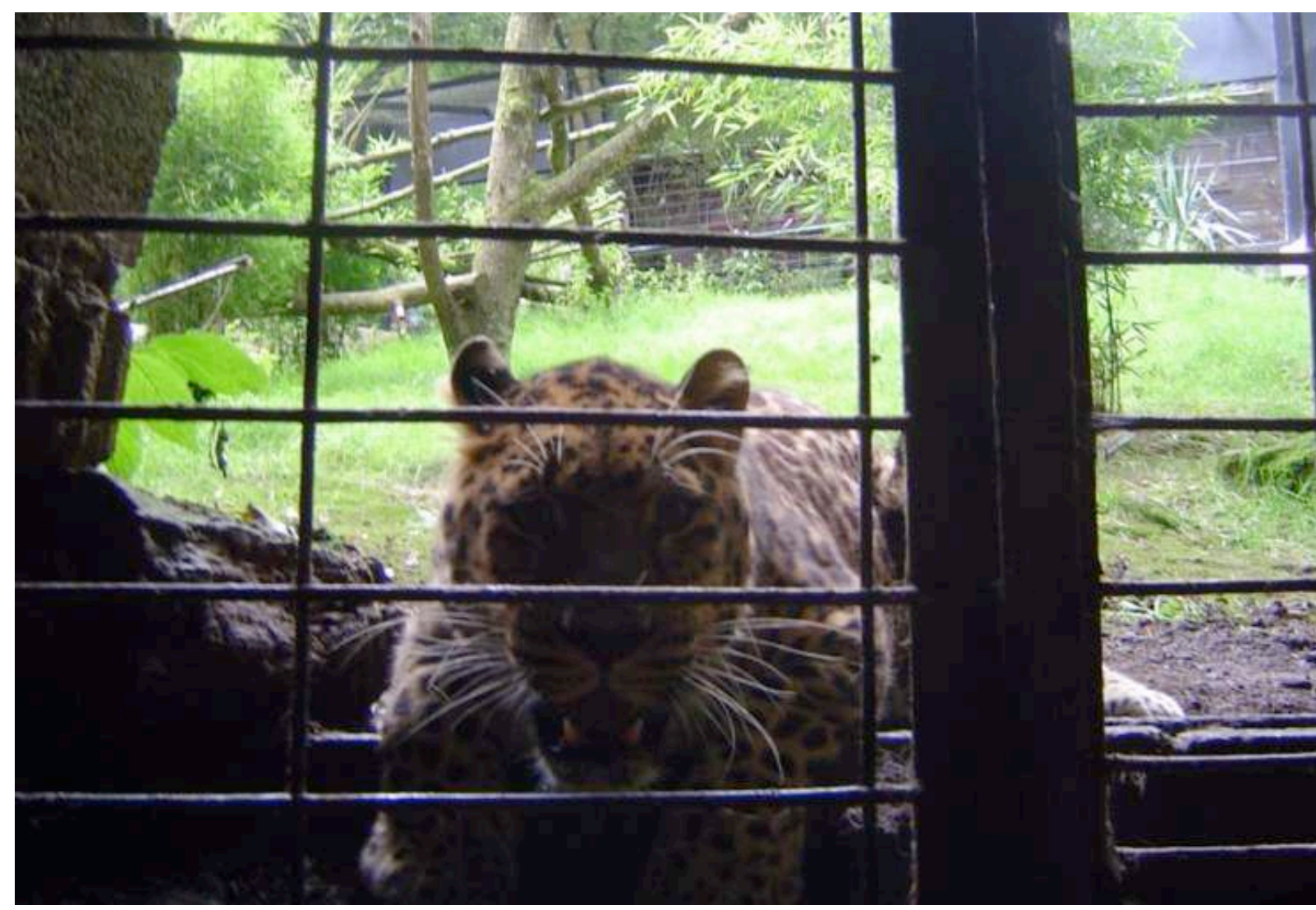

Figure 3 : La sale bête du zoo de Pont Scorff, 27 juillet 2007

Son corps est tendu vers la grille et elle rugit en me regardant. Manifestement, le jeu du balai ne l'intéresse plus. Je ne suis vraiment pas rassuré et à peine mon cliché pris, je sors du réduit. Cette sale bête est en train de me faire rater une partie de mon travail de la matinée, je n'ai pas posé les questions que je voulais à Stéphanie et me voici désormais condamné à l'attendre dans les allées du zoo. Décidément, le mieux que j'aie à faire est encore d'oublier l'épisode et d'être plus prudent à l'avenir.

C'est bien ce que je pense faire jusqu'au moment où, pour les besoins de ma thèse (Estebanez, 2010), je cherche à rendre compte de ce qu'est l'exotisme. Curieusement, cette scène me revient à l'esprit, non parce qu'elle illustrerait parfaitement le phénomène, mais au contraire, parce que je me rends compte qu'il en est totalement absent. C'est pourtant essentiel au zoo, l'exotisme (Estebanez, 2008).

Au moment où je vis la scène, la panthère est menaçante, effrayante, féroce même, mais pas exotique. II y a, dans l'exotisme, la transformation de quelque chose en objet de désir. Or ce qui m'effraye réellement, par exemple parce que je crains pour ma vie -et à la différence de la peur qu'on peut jouer à ressentir derrière une solide grille- ne peut pas être objet de désir, au moins sur le moment. Retravaillé dans une discussion avec des amis, par des photographies, dans un reportage ou dans un livre, l'épisode qui m'a pétrifié peut gagner en exotisme. II suffirait par exemple que je change le discours accompagnant les deux photographies, effaçant la réelle peur qui me saisit, insistant sur la majesté de l'animal, la chance que j'ai d'être aussi proche d'elle, mon courage manifeste, pour que son sens 
change. L'exotisme est un processus qui rend l'altérité attirante (Staszak, 2008 ; 2011), transformant objets, vivants et lieux, afin d'en réduire la radicalité.

Ce qui est en jeu dans cet épisode, c'est la possibilité d'une pratique sensible dans laquelle on peut penser à partir d'une expérience corporelle. Ici, au fond, j'apprends avec la vulnérabilité de mon propre corps, qui me permet de produire une critique de l'exotisme, sans passer par la déconstruction culturelle de la notion. L'exotique ne peut faire -vraimentpeur.

Partant de ce point, j'essaye de penser autrement ce qu'il s'est passé. En me faisant peur, la panthère $m^{\prime}$ a obligé à la penser autrement. Pour commencer, elle $m^{\prime}$ a forcé à la considérer. Simple arrière-plan de mon dispositif, elle faisait partie des animaux, cet élément générique abstrait qui m'intéresse essentiellement parce qu'il importe aux humains. Je fais des sciences sociales, après tout. Or, ce ne sont pas les animaux mais bien elle, avec sa présence singulière et menaçante qui me regarde et rugit. Comme je ne peux la mettre à l'écart, je suis obligé de sortir. Cette panthère ne se confondra plus avec aucune des dizaines d'autres panthères que j'ai croisées dans mes visites, elle est devenue la sale bête, pauvre qualification de ses compétences, mais qui l'individualise définitivement pour moi.

On peut considérer que c'est un fâcheux imprévu, qui ne fait que corrompre ou retarder le déroulement de mon protocole de recherche avec les soigneurs. On peut aussi considérer que c'est une chance. Alors que mon dispositif est bien établi, ce que j'apprends m'offre de moins en moins de possibilités de transformation des idées, et de changement de mes habitudes de recherche et de pensée. Avec la disparition du risque -je sais grosso modo ce que je vais apprendre- c'est aussi la possibilité d'être surpris par ce que j'apprends qui s'efface.

En s'imposant à moi, la panthère brise le dispositif appauvrissant -au moins à son égard- que je comptais déployer. Rien dans celui-ci qui puisse l'intéresser puisque je ne m'adresse pas à elle. Ce que je questionne, ce sont les visiteurs et le dispositif spectaculaire du zoo notamment sa capacité à modeler une image des animaux, qui deviennent sauvages et exotiques. Par contre, à propos des animaux singuliers (Lestel, 2004), rien. La panthère n'a ainsi pas vraiment d'occasion de faire preuve de ses compétences. À vrai dire, selon la belle formule de Vinciane Despret (2002), dans mon dispositif de terrain, je ne lui ai laissé aucune chance de me laisser une chance de saisir ses compétences.

Mais allons plus loin : $s^{\prime}$ il se passe quelque chose, c'est que la panthère me fait, elle, une proposition. J'ai certes dans l'instant l'impression qu'elle veut me dévorer -et j'ai du mal à penser qu'il s'agisse d'une bonne façon de faire connaissance- mais elle est, à la réflexion, parfaitement consciente qu'il y a entre elle et moi une grille, dont elle connait exactement les capacités. Après tout, elle habite ici depuis des années. Elle sait bien qu'il y a peu de chances que je fasse office d'amuse-gueule. Pour autant, elle s'adresse à moi, et quitte pour cela le jeu du balai.

$\mathrm{Si}$, malgré les apparences, il y a une certaine " politesse du faire connaissance " (Despret, 2002) dans la proposition de la panthère, c'est que je peux y prendre position et ainsi signaler si oui ou non elle m'intéresse. En sortant, je la refuse, mais en restant marqué par 
l'épisode, par ce que la panthère m'a fait faire, je lui laisse une chance d'agir, que je saisis bien après l'avoir vécu.

Au zoo, les visiteurs sollicitent continument les animaux qu'ils préfèrent par des cris, des gestes voire des jets de nourriture ou d'objets auxquels ceux-ci répondent le plus souvent par de l'indifférence, alors que le simple passage, au loin, de leur soigneur suffit parfois à faire bondir un lion dont on aurait juré qu'il dormait. Ne rien faire est une forme de réponse. Quand le chimpanzé Sarah (ibid.) refuse de former des phrases avec des lettres en plastique, comme elle sait habituellement le faire, c'est que les chercheurs qui s'occupent d'elle la laissent seule. Ce qui l'intéresse, ce n'est pas de parler, mais de parler avec quelqu'un. Si on peut sans doute par la contrainte empêcher quelqu'un de parler, on pourra difficilement lui permettre de développer de nouvelles compétences par les mêmes méthodes. Poser des questions polies, c'est intéresser ceux à qui on s'adresse, en leur offrant la possibilité de résister à la proposition, et en nous offrant ainsi la possibilité de transformer notre question.

Les animaux posent-ils des questions? On peut au moins avancer qu'ils font des propositions. Wattana (voir à son sujet Herzfeld, 2012), une des orangs-outangs de la Ménagerie du Jardin des Plantes de Paris, avait pour habitude de déchirer de grands sacs de jute déposés dans son enclos en longues lanières. Se hissant dans la partie haute de l'enclos, formée de barreaux, elle faisait pendre la lanière à l'extérieur de la cage, pour " pêcher le public ", d'après la description des soigneurs. Elle proposait ainsi au public de jouer avec elle, dans une version renouvelée du pompon des fêtes foraines qu'on décroche pour gagner un tour de manège gratuit.

Cette orang-outang -véritable surdouée, il est vrai (Herzfeld \& Lestel, 2005)- évite ainsi l'ennui en jouant avec la clôture du zoo et le public. Elle rend ainsi manifeste une fonction majeure du zoo, qui n'est pas tant de séparer les vivants humains et non humains, que d'organiser leur proximité. Il s'agit certes de voir les animaux, mais mieux encore de faire quelque chose avec eux. On restera quelques secondes devant un lion qui semble dormir, quelques minutes peut-être devant des babouins qui se toilettent mutuellement mais beaucoup plus si Wattana joue avec nous. Ce qui fascine les visiteurs qui assistent à la scène est d'ailleurs bien qu'elle soit à l'initiative de l'action, manifestant ainsi son autonomie et son goût pour la vie en commun.

La proposition que la panthère me fait est certes moins explicite mais elle s'adresse bien à moi et sollicite mon attention.

Certes -et bien que Wattana et ses cordes puisse nous faire douter- ce sont les animaux qui répondent à nos questions. Si les perroquets gris, dont on savait déjà qu'ils parlaient, comprennent ce qu'ils disent, expriment leur désirs et manipulent des catégories abstraites, c'est grâce aux travaux d'Irène Pepperberg (1995). Les moutons ne sont plus seulement des gigots en puissance depuis les travaux de Thelma Rowell (2001), qui ont montré qu'ils avaient de réelles compétences sociales. Les babouins qui vivaient dans une société rigidement hiérarchisée jusque dans les années 70, deviennent, dans les travaux de Strum et Fedigan (2001), des sociologues à fourrure, aux comportements flexibles et qui savent nourrir l'amitié des femelles afin d'accroitre leur influence. 
Les animaux ne peuvent toujours pas se passer de porte-parole humains pour acquérir une biographie plus riche. Pourtant, comme le signalent Isabelle Stengers (2011) et Vinciane Despret (2002), à sa suite, ce n'est pas parce qu'ils existent dans nos histoires qu'ils sont moins réels. Ces histoires où ils acquièrent de nouvelles compétences renvoient toujours à eux : ils en sont une condition de possibilité. Ce ne sont d'ailleurs pas des histoires sur eux, mais avec eux. Elles ne portent pas sur la transformation de l'un ou de l'autre (est-ce la panthère qui change ? Sont-ce mes représentations de la panthère ?) mais sur une existence commune pour laquelle il faut inventer, à chaque fois, des propositions spécifiques.

Si nous suivons la proposition de Dominique Lestel ${ }^{2}$, il est sans doute plus habile de ne pas poser la question sous sa forme ontologique - "qu'est-ce que l'autre ? » voire, dans une version appauvrissante, " en quoi l'autre est-il différent de moi ? »-, mais de se demander, de manière pragmatique : "que fait l'autre? ".

En passant dans le registre des pratiques, des résultats et des conséquences et donc, en premier lieu, du corps, la question devient « qu'est-ce que l'autre (ne) peut-il (pas) faire sur moi? " et inversement " qu'est-ce que je (ne) peux (pas) faire sur lui ? ". C'est bien d'ailleurs ce que je note alors : la panthère me fait quelque chose. Elle me pousse à quitter le réduit dans lequel j'étais, alors même que j'avais la ferme intention de suivre le travail de Stéphanie, et elle me force à la considérer, elle.

À la suite de cet épisode, mon travail se transforme, faisant émerger des choses qui étaient là -dans mes notes, mes photographies, mes souvenirs- mais que je n'avais pas vues. Les animaux acquièrent des compétences et la visite au zoo prend un intérêt neuf -même s'il est souvent déçu, tant on peut s'ennuyer, quand on est ignoré- autour de la vie en commun.

Cette capacité à agir sur les autres, qu'on peut nommer agentivité ${ }^{3}$, dessine un monde dans lequel, au gré des propositions saisies ou non, nous composons avec eux, sans qu'il soit d'abord question de nos représentations et des problématiques images mentales auxquelles elles renvoient.

S'intéresser aux communautés hybrides humains-non humains (Lestel, 2007 ; 2004) amène à penser le corps et avec lui. Bien loin de réactiver la dichotomie classique avec l'esprit, il s'agit plutôt de s'interroger sur une pensée débordant le cerveau, qui n'est plus son unique moteur, pour aussi se nourrir des lieux et des contextes. On peut proposer ici trois éléments pour imaginer ce qu'on peut faire du corps.

Par sa capacité à toujours réintroduire le trouble et la surprise, du fait de sa disponibilité et de sa vulnérabilité, le corps se présente comme un outil de recherche voire une exigence méthodologique.

\footnotetext{
${ }^{2}$ Voir l'entretien dans ce numéro, dans la rubrique Carnets de Débats.

${ }^{3}$ Le terme d'agency n'a pas de réel équivalent en français. Il est traduit, selon les auteurs, par capacité d'agir, puissance d'agir, agence, agentivité, agencéité... Après les travaux exploratoires de Michel Foucault et de Pierre Bourdieu, ce concept central dans les premières théories anglophones féministes à été assez largement repris en sciences sociales, au point qu'on à pu parler pour les années 1990 d'un agency turn (Hoskin in Tilley et al., 2006). Pour un aperçu sur la complexité du concept d'agency et son usage, en particulier dans les études de genre, champs dans lequel il est encore majoritairement mobilisé, on peut voir Montenach (2012).
} 
Le corps s'impose pour penser la vie en commun, qui ne passe pas que par le langage parlé et articulé - et ce, d'autant plus nettement qu'on s'intéresse aux communautés humanimales. C'est un élément de traduction majeur qui nous propose de suivre les pratiques et nous engage ainsi sur la voie d'une symétrie croissante des acteurs.

Enfin, en permettant une convergence avec les travaux sur la performance et la théorie plusque-représentationnelle, l'entrée par le corps permet aux études humanimales de ne pas être cantonnées dans une position de niche disciplinaire mais plutôt comme une façon de redéfinir la société voire ce qu'est l'existence.

\section{Bibliographie}

Despret V. (2002), Quand le loup habitera avec l'agneau, Paris, Les empêcheurs de penser en rond, $284 \mathrm{p}$.

Estebanez J. (2010), Les zoos comme dispositif spatial : pour une géographie culturelle de l'animalité, Thèse de doctorat, Paris 7/Université de Genève, $411 \mathrm{p}$.

Estebanez J. (2010) «Ceux qui sont proches : les soigneurs au zoo », Société, 108, 2, p. 47-57

Estebanez J. (2008) « Les jardins zoologiques ou l'exotique à portée de main », Le Globe, 2008, 148, p. 89-105.

Herzfeld C. (2012), Wattana. Un orang-outang à Paris, Paris, Payot, 288 p.

Herzfeld C. \& Lestel D. (2005) « Knot Tying in Great Apes. Etho-ethnology of an Unusual Tool Behavior ", Social Science Information, Vol. 44, N4, p. 621-653

Hoskins J. (2006) "Agency, Biography and Objects " in Tilley C., Keane W., Kuechler-Fogden S., Rowlands M., Spyer P. (eds.), Handbook of Material Culture, London, Sage, 576 p.

Lestel D. (2007), L'animalité, Paris, Les Cahiers de l'Herne, 125 p.

Lestel D. (2004), L'animal singulier, Paris, Seuil, 138 p.

Montenach A. (dir.) (2012) " Agency : un concept opératoire dans les études de genre ? ", numéro spécial de Rives Méditerranéennes, 41

Pepperberg I. (1995) « Grey Parrots Intelligence », Proceedings of the International

Aviculturists Society, january, p. 11-15

Rowell T. (2001) «A few peculiar Primates » in Strum S. \& Fedigan L., Primate Encounters: Models of Science, Gender and Society, Chicago, Chicago University Press, p. 57-71.

Stengers I. (2011), L'invention des sciences modernes, Paris, Flammarion, 207 p.

Strum S. \& Fedigan L. (2001), Primate Encounters: Models of Science, Gender and Society, Chicago, Chicago University Press, 636 p.

Staszak J.-F. (2011) "La Fabrique cinématographique de l'altérité », Annales de Géographie, 682,6 , pp. 577-603

Staszak J.-F. (2008) « Qu'est-ce que l'exotisme? », Le Globe, 148, pp. 7-30. 